TITLE:

\title{
Kernel approximation approach to the Llanalysis of sampled-data systems
}

$\operatorname{AUTHOR}(S)$ :

Kim, Jung Hoon; Hagiwara, Tomomichi

\section{CITATION:}

Kim, Jung Hoon ... [et al]. Kernel approximation approach to the Llanalysis of sampleddata systems. 2015 European Control Conference (ECC) 2015: 416-421

ISSUE DATE:

2015-07

URL:

http://hdl.handle.net/2433/235574

\section{RIGHT:}

(c) 2015 IEEE. Personal use of this material is permitted. Permission from IEEE must be obtained for all other uses, in any current or future media, including reprinting/republishing this material for advertising or promotional purposes,

creating new collective works, for resale or redistribution to servers or lists, or reuse of any copyrighted component of this work in other works.; This is not the published version. Please cite only the published version.; この論文は出版社版

でありません。引用の際には出版社版をご確認ご利用ください。 


\title{
Kernel Approximation Approach to the $L_{1}$ Analysis of Sampled-Data Systems
}

\author{
Jung Hoon Kim and Tomomichi Hagiwara
}

\begin{abstract}
This paper provides a method for the $L_{1}$ analysis of sampled-data systems, by which we mean the computation of their $L_{\infty}$-induced norm. We first apply the lifting approach to sampled-data systems and derive an operator theoretic representation of their input/output relation. We then apply fast-lifting by which the sampling interval $[0, h)$ is divided into $M$ subintervals with an equal width, and provide methods for computing the $L_{\infty}$-induced norm. Specifically, we use an idea of kernel approximation approach, in which the kernel function of an input operator and the hold function of an output operator are approximated by staircase or piecewise linear functions. Furthermore, it is shown that the approximation errors in staircase or piecewise linear approximation are ensured to be reciprocally proportional to $M$ or $M^{2}$, respectively.
\end{abstract}

\section{INTRODUCTION}

There have been a number of studies associated with sampled-data systems [1]-[19] taking account of their intersample behavior. These studies can be classified by the type of system norms dealt with, where the typical studies are the $H_{\infty}$ problem [2],[3],[8]-[11] and the $H_{2}$ problem [7]-[9], [12]-[14]. Even though $H_{\infty}$ and $H_{2}$ norms play important roles in the analysis and synthesis for sampled-data systems relevant to practical control problems, they cannot be used for dealing with the problems of bounded persistent disturbances. In this regard, the $L_{1}$ problem of sampled-data systems, which deals with the $L_{\infty}$-induced norm of sampleddata systems, should be considered. In [15]-[18],methods for computing the $L_{\infty}$-induced norm of sampled-data systems have been provided by using the idea of fast-sample/fast-hold (FSFH) approximation or input approximation approach. More precisely, in [15]-[17], a sampled-data system is "approximated" by a discrete-time system through FSFH approximation [4], and it is shown that the $l_{\infty}$-induced norm of the approximating discrete-time system converges to the $L_{\infty}$ induced norm of the original sampled-data systems as the FSFH approximation parameter $M$ tends to infinity. However, these studies do not evaluate how close the $l_{\infty}$-induced norm for a given $M$ is to the exact value of the $L_{\infty}$-induced norm. In contrast, [18] derives readily computable upper and lower bounds of the $L_{\infty}$-induced norm of sampleddata systems by using the ideas of fast-lifting [19] and the input approximation approach. The latter approach was first developed in a relevant problem in continuous-time [20] and employs a staircase approximation or piecewise linear approximation scheme. The technique called fast-lifting [19]

Jung Hoon Kim and Tomomichi Hagiwara are with the Department of Electrical Engineering, Kyoto University, Nishikyo-ku, Kyoto 615-8510, Japan. kimejaguar.kuee.kyoto-u.ac.jp, hagiwara@kuee.kyoto-u.ac.jp plays an important role in introducing these approximation schemes, which divides the sampling interval $[0, h)$ into $M$ subintervals with an equal width (without applying sampling of signals). For the fast-lifting parameter $M$, it is shown that the gap between the upper and lower bounds in the method of [18] converges to 0 at convergence rates of $1 / M$ and $1 / M^{2}$ in the staircase approximation scheme and the piecewise linear approximation scheme, respectively.

As a significant advance over the existing result, this paper further develops an alternative method for computing the upper and lower bounds of the $L_{\infty}$-induced norm of sampled-data systems by using the idea of kernel approximation approach [21]. This direction of arguments is stimulated by the success of employing the kernel approximation approach in [21] in computing the $L_{\infty}$-induced norm of continuous-time LTI systems. More precisely, it was shown in [21] that the kernel approximation approach leads to an improved method for computing upper and lower bounds of the $L_{\infty}$-induced norm than the existing input approximation approach [20], even though the mere 'qualitative assertion' on the associated convergence rates remains the same as that in the input approximation approach: the approximation errors converge to 0 at the rates of $1 / M$ and $1 / M^{2}$ in the staircase and piecewise linear approximation schemes, respectively. This paper shows that this advantage of the kernel approximation approach is inherited to the $L_{1}$ analysis of sampled-data systems by providing readily computable upper and lower bounds of the induced norm.

The organization of this paper is as follows. We first review sampled-data systems and the lifting approach to such systems in Section II. We next provide a computation method for the $L_{\infty}$-induced norm of sampled-data systems in Section III. More precisely, we apply the ideas of the fast-lifting [19] and the kernel approximation approach [21] to the $L_{\infty}$-induced norm analysis of sampled-data systems. By an adequate introduction of staircase approximation and piecewise linear approximation schemes, we show that the $L_{\infty}$-induced norm of sampled-data systems is approximated by the $\infty$-norm of a suitably constructed matrix in each scheme, and further give an upper bound and a lower bound of the $L_{\infty}$-induced norm that can be computed easily. We further show that the error stemming from the approximation treatment is ensured to converge to 0 at the rates of $1 / M$ and $1 / M^{2}$ in the staircase and piecewise linear approximation schemes, respectively.

In the following, the notations $\mathbb{N}, \mathbb{R}$ and $\mathcal{K}_{\nu}$ are used to mean the set of positive integers, the set of real numbers and $\left(L_{\infty}[0, h)\right)^{\nu}$, respectively. We further use the notation $\mathbb{N}_{0}$ to 
imply $\mathbb{N} \cup\{0\}$. The notation $\|\cdot\|$ is used to mean either the $L_{\infty}[0, h)$ norm of a matrix function, i.e.,

$$
\|F(\cdot)\|:=\max _{i} \sup _{0 \leq t<h} \sum_{j}\left|F_{i j}(t)\right|,
$$

the $L_{\infty}[0, h)$-induced norm of an operator (or these with $h$ replaced by $h / M$ ), or the $\infty$-norm of a finite-dimensional matrix, whose distinction will be clear from the context.

\section{LIFTED REPRESENTATION OF SAMPLED-DATA SYSTEMS}

Let us consider the stable sampled-data system $\Sigma_{\mathrm{SD}}$ shown in Fig. 1, where $P$ denotes the continuous-time LTI generalized plant, while $\Psi, \mathcal{H}$ and $\mathcal{S}$ denote the discrete-time LTI controller, the zero-order hold and the ideal sampler, respectively, operating with sampling period $h$ in a synchronous fashion. Solid lines and dashed lines in Fig. 1 are used to represent continuous-time signals and discrete-time signals, respectively. Suppose that $P$ and $\Psi$ are described respectively by

$$
\begin{gathered}
P:\left\{\begin{array}{l}
\frac{d x}{d t}=A x+B_{1} w+B_{2} u \\
z=C_{1} x+D_{11} w+D_{12} u \\
y=C_{2} x
\end{array}\right. \\
\Psi:\left\{\begin{array}{l}
\psi_{k+1}=A_{\Psi} \psi_{k}+B_{\Psi} y_{k} \\
u_{k}=C_{\Psi} \psi_{k}+D_{\Psi} y_{k}
\end{array}\right.
\end{gathered}
$$

where $x(t) \in \mathbb{R}^{n}, w(t) \in \mathbb{R}^{n_{w}}, u(t) \in \mathbb{R}^{n_{u}}, z(t) \in$ $\mathbb{R}^{n_{z}}, y(t) \in \mathbb{R}^{n_{y}}, \psi_{k} \in \mathbb{R}^{n_{\Psi}}, y_{k}=y(k h)$ and $u(t)=$ $u_{k}(k h \leq t<(k+1) h)$.

Because the sampled-data system $\Sigma_{\mathrm{SD}}$ is a hybrid continuous-time/discrete-time system, this system viewed in continuous-time is (periodically) time-varying. To deal with $\Sigma_{\mathrm{SD}}$ as a time-invariant system, we apply the lifting technique [1]-[3]. That is, given $f \in\left(L_{\infty}\right)^{\nu}$, its lifting $\left\{\widehat{f}_{k}\right\}_{k=0}^{\infty}$ with $\widehat{f}_{k} \in \mathcal{K}_{\nu}$ (with sampling period $h$ ) is defined as follows [1]-[3]:

$$
\widehat{f_{k}}(\theta)=f(k h+\theta) \quad(0 \leq \theta<h)
$$

By applying lifting to $w$ and $z$, the lifted representation of the sampled-data system $\Sigma_{\mathrm{SD}}$ is described by

$$
\left\{\begin{array}{l}
\xi_{k+1}=\mathcal{A} \xi_{k}+\mathcal{B} \widehat{w}_{k} \\
\widehat{z}_{k}=\mathcal{C} \xi_{k}+\mathcal{D} \widehat{w}_{k}
\end{array}\right.
$$

with $\xi_{k}:=\left[\begin{array}{ll}x_{k}^{T} & \psi_{k}^{T}\end{array}\right]^{T}\left(x_{k}:=x(k h)\right)$, the matrix

$$
\mathcal{A}=\left[\begin{array}{cc}
A_{d}+B_{2 d} D_{\Psi} C_{2 d} & B_{2 d} C_{\Psi} \\
B_{\psi} C_{2 d} & A_{\Psi}
\end{array}\right]: \mathbb{R}^{n+n_{\Psi}} \rightarrow \mathbb{R}^{n+n_{\Psi}}
$$

and the operators

$$
\begin{aligned}
\mathcal{B} & =J_{\Sigma} \mathbf{B}_{1}: \mathcal{K}_{n_{w}} \rightarrow \mathbb{R}^{n+n_{\Psi}} \\
\mathcal{C} & =\mathbf{M}_{1} C_{\Sigma}: \mathbb{R}^{n+n_{\Psi}} \rightarrow \mathcal{K}_{n_{z}} \\
\mathcal{D} & =\mathbf{D}_{11}: \mathcal{K}_{n_{w}} \rightarrow \mathcal{K}_{n_{z}}
\end{aligned}
$$

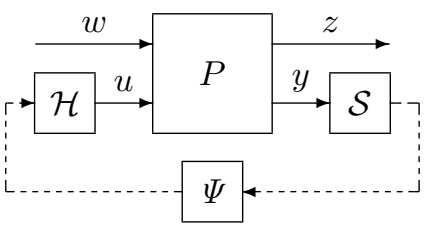

Fig. 1. Sampled-data system $\Sigma_{\mathrm{SD}}$.

where

$$
\begin{aligned}
& A_{d}:=\exp (A h), B_{2 d}:=\int_{0}^{h} \exp (A \theta) B_{2} d \theta, C_{2 d}:=C_{2} \\
& J_{\Sigma}:=\left[\begin{array}{l}
I \\
0
\end{array}\right] \in \mathbb{R}^{\left(n+n_{\Psi}\right) \times n}, C_{\Sigma}:=\left[\begin{array}{cc}
I & 0 \\
D_{\Psi} C_{2 d} & C_{\Psi}
\end{array}\right] \\
& \mathbf{B}_{1} w=\int_{0}^{h} \exp (A(h-\theta)) B_{1} w(\theta) d \theta \\
& \left(\mathbf{M}_{1}\left[\begin{array}{l}
x \\
u
\end{array}\right]\right)(\theta)=C_{0} \exp \left(A_{2} \theta\right)\left[\begin{array}{l}
x \\
u
\end{array}\right] \\
& A_{2}:=\left[\begin{array}{cc}
A & B_{2} \\
0 & 0
\end{array}\right], C_{0}:=\left[\begin{array}{ll}
C_{1} & D_{12}
\end{array}\right] \\
& \left(\mathbf{D}_{11} w\right)(\theta)=\int_{0}^{\theta} C_{1} \exp (A(\theta-\tau)) B_{1} w(\tau) d \tau+D_{11} w\left(\theta^{\prime}\right)
\end{aligned}
$$

From the stability assumption of $\Sigma_{\mathrm{SD}}, \mathcal{A}$ is stable, i.e., has all its eigenvalues in the open unit disc.

\section{COMPutation OF The $L_{\infty}$-INDUCED Norm OF} SAMPLED-DATA SySTEMS VIA KERNEL APPROXIMATION

This section gives the main results of this paper, i.e., two methods for computing the $L_{\infty}$-induced norm of $\Sigma_{\mathrm{SD}}$ (more precisely, its upper and lower bounds converging to each other as the fast-lifting parameter $M$ tends to $\infty$ ) by introducing the kernel approximation approach with staircase approximation and piecewise linear approximation schemes. Furthermore, the associated convergence rates are also given. Here, for $M \in \mathbb{N}$ and $h^{\prime}:=h / M$, fastlifting [19] is defined as the mapping from $f \in \mathcal{K}_{\nu}$ to $\check{f}:=\left[\begin{array}{lll}\left(f^{(1)}\right)^{T} & \cdots & \left(f^{(M)}\right)^{T}\end{array}\right]^{T} \in\left(\mathcal{K}_{\nu}^{\prime}\right)^{M}$, and is denoted by $\check{f}=\mathbf{L}_{M} f$, where

$$
f^{(i)}\left(\theta^{\prime}\right):=f\left((i-1) h^{\prime}+\theta^{\prime}\right) \quad\left(0 \leq \theta^{\prime}<h^{\prime}\right)
$$

and $\mathcal{K}_{\nu}^{\prime}$ is a shorthand notation for $\left(L_{\infty}\left[0, h^{\prime}\right)\right)^{\nu}$. It is easy to see that $\mathbf{L}_{M}$ is norm-preserving (i.e., $\left\|\mathbf{L}_{M} f\right\|=\|f\|$ ), which plays a crucial role in the following arguments.

\section{A. Toeplitz Structure of Input/Output Relation and Trunca- tion}

To compute the $L_{\infty}$-induced norm of $\Sigma_{\mathrm{SD}}$, we first note (5) and describe the closed-loop relation between $\widehat{w}_{k}$ and 
$\widehat{z}_{k}(k=0, \cdots, \infty)$ as follows:

$\left[\begin{array}{c}\widehat{z}_{0} \\ \widehat{z}_{1} \\ \widehat{z}_{2} \\ \widehat{z}_{3} \\ \vdots\end{array}\right]=\left[\begin{array}{cccccc}\mathcal{D} & 0 & \ldots & & & \\ \mathcal{C B} & \mathcal{D} & 0 & \ldots & & \\ \mathcal{C} \mathcal{A B} & \mathcal{C B} & \mathcal{D} & 0 & \ldots & \\ \mathcal{C} \mathcal{A}^{2} \mathcal{B} & \mathcal{C} \mathcal{A B} & \mathcal{C B} & \mathcal{D} & 0 & \ldots \\ \vdots & \ddots & \ddots & \ddots & \ddots & \ddots\end{array}\right]\left[\begin{array}{c}\widehat{w}_{0} \\ \widehat{w}_{1} \\ \widehat{w}_{2} \\ \widehat{w}_{3} \\ \vdots\end{array}\right]$

Since the above operator has a Toeplitz structure, it follows readily that the $L_{\infty}$-induced norm of $\Sigma_{\mathrm{SD}}$ coincides with the $L_{\infty}$-induced norm of its "last" block row, i.e., (after reordering without affecting the $L_{\infty}$-induced norm)

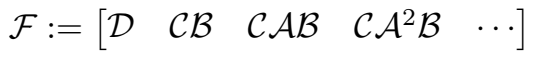

It is, however, still difficult to compute $\|\mathcal{F}\|$ since $\mathcal{F}$ consists of an infinite number of columns. To alleviate this difficulty, we first take an $N \in \mathbb{N}$, decompose $\mathcal{F}$ into

$$
\mathcal{F}=\mathcal{F}_{N}^{-}+\mathcal{F}_{N}^{+}
$$

where

$$
\begin{aligned}
\mathcal{F}_{N}^{-} & :=\left[\begin{array}{llllll}
\mathcal{D} & \cdots & \mathcal{C A}^{N} \mathcal{B} & 0 & 0 & \cdots
\end{array}\right] \\
\mathcal{F}_{N}^{+} & :=\left[\begin{array}{llllll}
0 & \cdots & 0 & \mathcal{C} \mathcal{A}^{N+1} \mathcal{B} & \mathcal{C A}^{N+2} \mathcal{B} & \cdots
\end{array}\right]
\end{aligned}
$$

and compute the $L_{\infty}$-induced norm $\left\|\mathcal{F}_{N}^{-}\right\|$as accurately as possible while the computation of $\left\|\mathcal{F}_{N}^{+}\right\|$is treated in a comparatively simple way (because this norm is expected to be small when $N$ is large enough); we aim at computing upper and lower bounds of $\|\mathcal{F}\|$ through approximation of $\mathcal{F}_{N}^{-}$and computing an upper bound of $\left\|\mathcal{F}_{N}^{+}\right\|$.

\section{B. Fast-lifting treatment of $\mathcal{F}_{N}^{-}$}

In this subsection, we review the fast-lifting treatment of $\mathcal{F}_{N}^{-}$. It immediately follows from the norm-preserving property of $\mathbf{L}_{M}$ that

$$
\left\|\mathcal{F}_{N}^{-}\right\|=\left\|\left[\mathbf{L}_{M} \mathcal{D} \mathbf{L}_{M}^{-1} \quad \cdots \quad \mathbf{L}_{M} \mathcal{C} \mathcal{A}^{N} \mathcal{B} \mathbf{L}_{M}^{-1}\right]\right\|
$$

To facilitate the treatment of the right-hand side, we introduce $\mathbf{D}_{11}^{\prime}, \mathbf{B}_{1}^{\prime}$ and $\mathbf{M}_{1}^{\prime}$ defined as $\mathbf{D}_{11}, \mathbf{B}_{1}$ and $\mathbf{M}_{1}$, respectively, with the horizon $[0, h)$ replaced by $\left[0, h^{\prime}\right)(=$ $[0, h / M))$, and also introduce the matrices

$$
\begin{aligned}
& A_{d}^{\prime}:=\exp \left(A h^{\prime}\right), A_{2 d}^{\prime}:=\exp \left(A_{2} h^{\prime}\right), \\
& J:=\left[\begin{array}{l}
I \\
0
\end{array}\right] \in \mathbb{R}^{\left(n+n_{u}\right) \times n}
\end{aligned}
$$

Then, (as in the standard arguments employing fastlifting, e.g., [19]), it is easy to see that $\mathbf{L}_{M} \mathcal{D} \mathbf{L}_{M}^{-1}$ and $\mathbf{L}_{M} \mathcal{C} \mathcal{A}^{j} \mathcal{B} \mathbf{L}_{M}^{-1}(j=0, \cdots, N)$ in (22) are described respectively by

$$
\begin{aligned}
& \mathbf{L}_{M} \mathcal{D} \mathbf{L}_{M}^{-1}=\overline{\mathbf{M}_{1}^{\prime}} \Delta_{M 0} \overline{\mathbf{B}_{1}^{\prime}}+\overline{\mathbf{D}_{11}^{\prime}} \\
& \mathbf{L}_{M} \mathcal{C} \mathcal{A}^{j} \mathcal{B} \mathbf{L}_{M}^{-1}=\overline{\mathbf{M}_{1}^{\prime}} A_{2 d M}^{\prime} C_{\Sigma} \mathcal{A}^{j} J_{\Sigma} A_{d M}^{\prime} \overline{\mathbf{B}_{1}^{\prime}}
\end{aligned}
$$

where

$$
\begin{gathered}
A_{d M}^{\prime}:=\left[\left(A_{d}^{\prime}\right)^{M-1} \quad \cdots I\right], A_{2 d M}^{\prime}:=\left[\begin{array}{c}
I \\
\vdots \\
\left(A_{2 d}^{\prime}\right)^{M-1}
\end{array}\right] \\
\Delta_{M 0}:=\left[\begin{array}{cccc}
0 & 0 & \cdots & 0 \\
J & \ddots & \ddots & \vdots \\
\vdots & \ddots & \ddots & 0 \\
\left(A_{2 d}^{\prime}\right)^{M-2} J & \cdots & J & 0
\end{array}\right]
\end{gathered}
$$

and $\overline{(\cdot)}$ denotes $\operatorname{diag}[(\cdot), \cdots,(\cdot)]$ consisting of $M$ copies of $(\cdot)$. Hence, the operator matrix on the right hand side of (22) admits the representation

$$
\begin{aligned}
& \mathcal{F}_{N M}^{-} \\
& \quad=\left[\begin{array}{lllll}
\overline{\mathbf{M}_{1}^{\prime}} \Delta_{M 0} \overline{\mathbf{B}_{1}^{\prime}}+\overline{\mathbf{D}_{11}^{\prime}} & \overline{\mathbf{M}_{1}^{\prime}} \mathcal{A}_{M 0} \overline{\mathbf{B}_{1}^{\prime}} & \ldots & \overline{\mathbf{M}_{1}^{\prime}} \mathcal{A}_{M N} \overline{\mathbf{B}_{1}^{\prime}}
\end{array}\right]
\end{aligned}
$$

where

$$
\mathcal{A}_{M j}:=A_{2 d M}^{\prime} C_{\Sigma} \mathcal{A}^{j} J_{\Sigma} A_{d M}^{\prime} \quad(j=0, \cdots, N)
$$

\section{Kernel approximation approach to $\mathcal{F}_{N M}^{-}$}

This subsection develops a framework for computing $\left\|\mathcal{F}_{N M}^{-}\right\|\left(=\left\|\mathcal{F}_{N}^{-}\right\|\right)$by using an idea of kernel approximation approach, which was introduced first in [21] to compute the $L_{\infty}$-induced norm of continuous-time LTI systems. In [21], it was shown that the kernel approximation approach leads to an improved method for computing upper and lower bounds of the $L_{\infty}$-induced norm than the existing input approximation approach [20]. Motivated by this achievement, this paper is interested in computing the $L_{\infty}$-induced norm of sampleddata systems through the idea of the kernel approximation approach. More precisely, we consider constant and linear approximations to the kernel function $\exp \left(A\left(h^{\prime}-\theta^{\prime}\right)\right) B_{1}$ of $\mathbf{B}_{1}^{\prime}$ together with constant and linear approximations of the 'hold function' $C_{0} \exp \left(A_{2} \theta^{\prime}\right)$ of $\mathbf{M}_{1}^{\prime}$, which respectively lead to staircase and piecewise linear approximations of signals if they are viewed on $[0, h)$ rather than $\left[0, h^{\prime}\right)$. Furthermore, we aim at showing the associated convergence rates in the fast-lifting parameter $M$.

1) Staircase approximation: We introduce the operators $\mathbf{B}_{\mathrm{k} 0}^{\prime}: \mathcal{K}_{n_{w}}^{\prime} \rightarrow \mathbb{R}^{n}[21], \mathbf{M}_{\mathrm{p} 0}^{\prime}: \mathbb{R}^{n+n_{u}} \rightarrow \mathcal{K}_{n_{z}}^{\prime}$ and $\mathbf{D}_{\mathrm{p} 0}^{\prime}:$ $\mathcal{K}_{n_{w}}^{\prime} \rightarrow \mathcal{K}_{n_{z}}^{\prime}[18]$ defined respectively as

$$
\begin{aligned}
& \mathbf{B}_{\mathrm{k} 0}^{\prime} w=\int_{0}^{h^{\prime}} A_{d}^{\prime} B_{1} w\left(\theta^{\prime}\right) d \theta^{\prime} \\
& \left(\mathbf{M}_{\mathrm{p} 0}^{\prime}\left[\begin{array}{l}
x \\
u
\end{array}\right]\right)\left(\theta^{\prime}\right)=C_{0}\left[\begin{array}{l}
x \\
u
\end{array}\right] \quad\left(0 \leq \theta^{\prime}<h^{\prime}\right) \\
& \left(\mathbf{D}_{\mathrm{p} 0}^{\prime} w\right)\left(\theta^{\prime}\right)=D_{11} w\left(\theta^{\prime}\right)
\end{aligned}
$$

Introducing the operator $\mathbf{B}_{\mathrm{k} 0}^{\prime}$ corresponds to the zeroorder approximation of the kernel function $\exp \left(A\left(h^{\prime}-\right.\right.$ $\left.\left.\theta^{\prime}\right)\right) B_{1}=A_{d}^{\prime} \sum_{i=0}^{\infty} \frac{\left(-A \theta^{\prime}\right)^{i}}{i !} B_{1}$ of the operator $\mathbf{B}_{1}^{\prime} \cdot \mathbf{M}_{\mathrm{p} 0}^{\prime}$ corresponds to the zero-order approximation of the hold 
function $C_{0} \exp \left(A_{w} \theta^{\prime}\right)$ of the operator $\mathbf{M}_{1}^{\prime}$, i.e., the zeroorder approximation of the Taylor expansion of the output of $\mathbf{M}_{1}^{\prime} . \mathbf{D}_{\mathrm{p} 0}^{\prime}$ means the operator of multiplication by the matrix $D_{11}$.

Remark 1: The approximation operators $\mathbf{M}_{\mathrm{p} 0}^{\prime}$ and $\mathbf{D}_{\mathrm{p} 0}^{\prime}$ are also used in [18] employing the input approximation approach, and the only difference between the kernel approximation approach and the input approximation approach [18] is the way the operator $\mathbf{B}_{1}^{\prime}$ is approximated. This is because the operators $\mathbf{M}_{1}^{\prime}$ and $\mathbf{D}_{11}^{\prime}$ are comparatively simple and thus there seem to be little variations for their reasonable approximations. Hence, the main contribution of the present paper over the existing results [18] is a new approximation approach of the operator $\mathbf{B}_{1}^{\prime}$. Essentially the same comments apply to the following arguments with the piecewise linear approximation scheme. As it turns out (see the last paragraph of Subsection III-D), the new method for approximating the operator $\mathbf{B}_{1}^{\prime}$ in this paper leads to an improved method for computing upper and lower bounds of the $L_{\infty}$-induced norm than the existing input approximation approach [18].

To proceed with the kernel approximation approach with the staircase approximation scheme, we consider the operator $\mathcal{F}_{N M 0}^{-}$obtained by replacing $\mathbf{B}_{1}^{\prime}, \mathbf{M}_{1}^{\prime}$ and $\mathbf{D}_{11}^{\prime}$ with $\mathbf{B}_{\mathrm{k} 0}^{\prime}, \mathbf{M}_{\mathrm{p} 0}^{\prime}$ and $\mathbf{D}_{\mathrm{p} 0}^{\prime}$, respectively, in (28):

$$
\begin{aligned}
& \mathcal{F}_{N M \mathrm{k} 0}^{-} \\
= & {\left[\begin{array}{lllll}
\overline{\mathbf{M}_{\mathrm{p} 0}^{\prime}} \Delta_{M 0} \overline{\mathbf{B}_{\mathrm{k} 0}^{\prime}}+\overline{\mathbf{D}_{\mathrm{p} 0}^{\prime}} & \overline{\mathbf{M}_{\mathrm{p} 0}^{\prime}} \mathcal{A}_{M 0} \overline{\mathbf{B}_{\mathrm{k} 0}^{\prime}} & \cdots & \overline{\mathbf{M}_{\mathrm{p} 0}^{\prime}} \mathcal{A}_{M N} \overline{\mathbf{B}_{\mathrm{k} 0}^{\prime}}
\end{array}\right] }
\end{aligned}
$$

This paper shows that $\left\|\mathcal{F}_{N M \mathrm{k} 0}^{-}\right\|$can be computed exactly and tends to $\left\|\mathcal{F}_{N}^{-}\right\|$as $M$ tends to infinity at the convergence rate of $1 / M$. The following two lemmas play important roles in establishing the latter fact.

Lemma 1: The inequality

$$
\left\|\mathbf{L}_{M} \mathcal{D} \mathbf{L}_{M}^{-1}-\left(\overline{\mathbf{M}_{\mathrm{p} 0}^{\prime}} \Delta_{M 0} \overline{\mathbf{B}_{\mathrm{k} 0}^{\prime}}+\overline{\mathbf{D}_{\mathrm{p} 0}^{\prime}}\right)\right\| \leq \frac{K_{M \mathcal{D k} 0}}{M}
$$

holds with $K_{M \mathcal{D} \mathrm{k} 0}$ defined as

$$
\begin{array}{r}
K_{M \mathcal{D} \mathrm{k} 0}:=h\left\|C_{1}\right\| \cdot\left\|B_{1}\right\| e^{\|A\| h / M}+\frac{h^{2}}{M}\|A\| \cdot\left\|B_{1}\right\| e^{\|A\| h / M} \\
\cdot \sum_{k=0}^{M-2}\left(\frac{1}{2}\left\|C_{1}\left(A_{d}^{\prime}\right)^{k+1}\right\|+\left\|C_{1}\left(A_{d}^{\prime}\right)^{k}\right\| e^{\|A\| h / M}\right)
\end{array}
$$

Furthermore, $K_{M \mathcal{D} \text { k0 }}$ has a uniform upper bound with respect to $M$ given by

$$
\begin{aligned}
K_{\mathcal{D k} 0}^{U} & :=h\left\|C_{1}\right\| \cdot\left\|B_{1}\right\| e^{\|A\| h} \\
& +h^{2}\left\|C_{1}\right\| \cdot\|A\| \cdot\left\|B_{1}\right\| e^{2\|A\| h}\left(\frac{1}{2}+e^{\|A\| h}\right)
\end{aligned}
$$

Lemma 2: The inequality

$$
\left\|\overline{\mathbf{M}_{1}^{\prime}} \mathcal{A}_{M j} \overline{\mathbf{B}_{1}^{\prime}}-\overline{\mathbf{M}_{\mathrm{p} 0}^{\prime}} \mathcal{A}_{M j} \overline{\mathbf{B}_{\mathrm{k} 0}^{\prime}}\right\| \leq \frac{K_{M j \mathrm{k} 0}}{M}
$$

holds for $j=0, \cdots, N$, where

$$
\begin{aligned}
& K_{M j \mathrm{k} 0}:=e^{\|A\| h / M}\left\|\mathcal{A}_{M j}\right\| \frac{h^{2}}{M} \\
& \cdot\left\{\left\|C_{0} A_{2}\right\| \cdot\left\|B_{1}\right\| e^{\left\|A_{2}\right\| h / M}+\frac{1}{2}\left\|C_{0}\right\| \cdot\|A\| \cdot\left\|A_{d}^{\prime} B_{1}\right\|\right\}
\end{aligned}
$$

Furthermore, $K_{M j \mathrm{k} 0}$ has a uniform upper bound with respect to $M$ and $j$ given by

$$
\begin{aligned}
K_{\mathcal{C} \mathcal{A B k} 0}^{U}: & =h^{2} e^{\|A\| h} \cdot\left\|B_{1}\right\| \cdot K_{*} \\
\cdot & \left\{\left\|C_{0} A_{2}\right\| e^{\left\|A_{2}\right\| h}+\frac{1}{2}\left\|C_{0}\right\| \cdot\|A\| e^{\|A\| h}\right\}
\end{aligned}
$$

where $K_{*}:=\max _{i \in \mathbb{N}_{0}}\left\|\mathcal{A}^{i}\right\| \cdot e^{\left(\|A\|+\left\|A_{2}\right\|\right) h} \cdot\left\|C_{\Sigma}\right\|$.

Lemmas 1 and 2 readily lead to the following result.

Proposition 1: The inequality

$$
\left\|\mathcal{F}_{N M}^{-}-\mathcal{F}_{N M \mathrm{k} 0}^{-}\right\| \leq \frac{K_{M \mathrm{k} 0}}{M}
$$

holds where

$$
K_{M \mathrm{k} 0}:=K_{M \mathcal{D} \mathrm{k} 0}+\sum_{j=0}^{N} K_{M j \mathrm{k} 0}
$$

In addition, $K_{M \mathrm{k} 0}$ has a uniform upper bound with respect to $M$ given by

$$
K_{\mathrm{k} 0}^{U}:=K_{\mathcal{D k} 0}^{U}+(N+1) \cdot K_{\mathcal{C} A \mathcal{B} \mathrm{k} 0}^{U}
$$

To evaluate $\left\|\mathcal{F}_{N}^{-}\right\|=\left\|\mathcal{F}_{N M}^{-}\right\|$through (40) and the triangle inequality, we next provide a method for (exactly) computing $\left\|\mathcal{F}_{N M \mathrm{k} 0}^{-}\right\|$. By essentially the same arguments as those in [18],[21], we can obtain the following result.

Proposition 2: $\left\|\mathcal{F}_{N M \mathrm{k} 0}^{-}\right\|$coincides with the $\infty$-norm of the finite-dimensional matrix $F_{N M \mathrm{k} 0}^{-}$given by

$$
\begin{aligned}
& F_{N M \mathrm{k} 0}^{-}:=\left[\begin{array}{llll}
\overline{D_{11}} & \overline{C_{0}} \Delta_{M 0} \overline{A_{d}^{\prime} B_{1} h^{\prime}} & \overline{C_{0}} \mathcal{A}_{M 0} \overline{A_{d}^{\prime} B_{1} h^{\prime}}
\end{array}\right. \\
& \text {... } \left.\overline{C_{0}} \mathcal{A}_{M N} \overline{A_{d}^{\prime} B_{1} h^{\prime}}\right]
\end{aligned}
$$

Combining the above propositions leads to the following first main result in this paper.

Theorem 1: The following inequality holds:

$$
\left\|F_{N M \mathrm{k} 0}^{-}\right\|-\frac{K_{M \mathrm{k} 0}}{M} \leq\left\|\mathcal{F}_{N}^{-}\right\| \leq\left\|F_{N M \mathrm{k} 0}^{-}\right\|+\frac{K_{M \mathrm{k} 0}}{M}
$$

This implies that upper and lower bounds of $\left\|\mathcal{F}_{N}^{-}\right\|$can be obtained through $\left\|F_{N M \mathrm{k} 0}^{-}\right\|$together with $K_{M \mathrm{k} 0} / M$, and by taking the fast-lifting parameter $M$ larger, the gap between those upper and lower bounds converges to 0 at no slower convergence rate than $1 / M$ (because $K_{M \mathrm{k} 0}$ has a uniform upper bound $\left.K_{\mathrm{k} 0}^{U}\right)$.

2) Piecewise linear approximation: We introduce the operators $\mathbf{B}_{\mathrm{k} 1}^{\prime}: \mathcal{K}_{n_{w}}^{\prime} \rightarrow \mathbb{R}^{n}[21], \mathbf{M}_{\mathrm{p} 1}^{\prime}: \mathbb{R}^{n+n_{u}} \rightarrow \mathcal{K}_{n_{z}}^{\prime}$ and $\mathbf{D}_{\mathrm{p} 1}^{\prime}: \mathcal{K}_{n_{w}}^{\prime} \rightarrow \mathcal{K}_{n_{z}}^{\prime}[18]$ defined respectively as

$$
\begin{aligned}
& \mathbf{B}_{\mathrm{k} 1}^{\prime} w=\int_{0}^{h^{\prime}} A_{d}^{\prime}\left(I-A \theta^{\prime}\right) B_{1} w\left(\theta^{\prime}\right) d \theta^{\prime} \\
& \left(\mathbf{M}_{\mathrm{p} 1}^{\prime}\left[\begin{array}{l}
x \\
u
\end{array}\right]\right)\left(\theta^{\prime}\right)=C_{0}\left(I+A_{2} \theta^{\prime}\right)\left[\begin{array}{l}
x \\
u
\end{array}\right] \quad\left(0 \leq \theta^{\prime}<h^{\prime}\right)
\end{aligned}
$$

$\left(\mathbf{D}_{\mathrm{p} 1}^{\prime} w\right)\left(\theta^{\prime}\right)=\int_{0}^{\theta^{\prime}} C_{1} B_{1} w\left(\theta^{\prime}\right) d \theta^{\prime}+D_{11} w\left(\theta^{\prime}\right)$ 
Introducing the operator $\mathbf{B}_{\mathrm{k} 1}^{\prime}$ corresponds to the first-order approximation of the kernel function of the operator $\mathbf{B}_{1}^{\prime}$. Introducing the operators $\mathbf{M}_{\mathrm{p} 1}^{\prime}$ and $\mathbf{D}_{\mathrm{p} 1}^{\prime}$ corresponds to the first-order approximation of the hold function of $\mathbf{M}_{1}^{\prime}$ and the kernel function of the 'compact part' of $\mathbf{D}_{11}^{\prime}$, respectively.

To proceed with the approximation arguments, we consider the operator $\mathcal{F}_{N M 1}^{-}$obtained by replacing $\mathbf{B}_{1}^{\prime}, \mathbf{M}_{1}^{\prime}$ and $\mathbf{D}_{11}^{\prime}$ with $\mathbf{B}_{\mathrm{k} 1}^{\prime}, \mathbf{M}_{\mathrm{p} 1}^{\prime}$ and $\mathbf{D}_{\mathrm{p} 1}^{\prime}$, respectively, in (28):

$$
\begin{aligned}
& \mathcal{F}_{N M \mathrm{k} 1}^{-} \\
= & {\left[\begin{array}{llll}
\overline{\mathbf{M}_{\mathrm{p} 1}^{\prime}} \Delta_{M 0} \overline{\mathbf{B}_{\mathrm{k} 1}^{\prime}}+\overline{\mathbf{D}_{\mathrm{p} 1}^{\prime}} & \overline{\mathbf{M}_{\mathrm{p} 1}^{\prime}} \mathcal{A}_{M 0} \overline{\mathbf{B}_{\mathrm{k} 1}^{\prime}} & \cdots & \overline{\mathbf{M}_{\mathrm{p} 1}^{\prime}} \mathcal{A}_{M N} \overline{\mathbf{B}_{\mathrm{k} 1}^{\prime}}
\end{array}\right] }
\end{aligned}
$$

Another main result of this paper is that $\left\|\mathcal{F}_{N M \mathrm{k} 1}^{-}\right\|$can be computed exactly and converges to $\left\|\mathcal{F}_{N}^{-}\right\|$at the rate of $1 / M^{2}$. The following two lemmas are important in establishing the latter fact.

Lemma 3: The inequality

$$
\left\|\mathbf{L}_{M} \mathcal{D} \mathbf{L}_{M}^{-1}-\left(\overline{\mathbf{M}_{\mathrm{p} 1}^{\prime}} \Delta_{M 0} \overline{\mathbf{B}_{\mathrm{k} 1}^{\prime}}+\overline{\mathbf{D}_{\mathrm{p} 1}^{\prime}}\right)\right\| \leq \frac{K_{M \mathcal{D k} 1}}{M^{2}}
$$

holds with $K_{M \mathcal{D} \mathrm{k} 1}$ defined as

$$
\begin{aligned}
K_{M \mathcal{D k} 1} & :=\frac{1}{2}\left\|C_{1}\right\| \cdot\|A\| \cdot\left\|B_{1}\right\| h^{2} e^{\|A\| h / M} \\
+\frac{1}{2}\|A\|^{2} \cdot\left\|B_{1}\right\| e^{\|A\| h / M} \frac{h^{3}}{M} \cdot \sum_{k=0}^{M-2}\left\{\left\|C_{1}\left(A_{d}^{\prime}\right)^{k}\right\| e^{\|A\| h / M}\right. & \\
& \left.+\frac{1}{3} \sup _{0 \leq \theta^{\prime}<h^{\prime}}\left\|C_{1}\left(I+A \theta^{\prime}\right)\left(A_{d}^{\prime}\right)^{k+1}\right\|\right\}
\end{aligned}
$$

Furthermore, $K_{M \mathcal{D k} 1}$ has a uniform upper bound with respect to $M$ given by

$$
\begin{aligned}
& K_{\mathcal{D} \mathrm{k} 1}^{U}:=\frac{1}{2}\left\|C_{1}\right\| \cdot\|A\| \cdot\left\|B_{1}\right\| h^{2} e^{\|A\| h} \\
& +\frac{1}{2}\left\|C_{1}\right\| \cdot\|A\|^{2} \cdot\left\|B_{1}\right\| e^{2\|A\| h} h^{3}\left(e^{\|A\| h}+\frac{1}{3}(1+\|A\| h)\right)
\end{aligned}
$$

Lemma 4: The inequality

$$
\left\|\overline{\mathbf{M}_{1}^{\prime}} \mathcal{A}_{M j} \overline{\mathbf{B}_{1}^{\prime}}-\overline{\mathbf{M}_{\mathrm{p} 1}^{\prime}} \mathcal{A}_{M j} \overline{\mathbf{B}_{\mathrm{k} 1}^{\prime}}\right\| \leq \frac{K_{M j \mathrm{k} 1}}{M^{2}}
$$

holds for $j=0, \cdots, N$, where

$$
\begin{aligned}
K_{M j \mathrm{k} 1}= & \frac{1}{2} e^{\|A\| h / M}\left\|\mathcal{A}_{M j}\right\| \frac{h^{3}}{M} \\
& \cdot\left\{\frac{1}{3} \sup _{0 \leq \theta^{\prime}<h^{\prime}}\left\|C_{0}\left(I+A_{2} \theta^{\prime}\right)\right\| \cdot\|A\|^{2} \cdot\left\|A_{d}^{\prime} B_{1}\right\|\right. \\
& \left.+\left\|C_{0} A_{2}^{2}\right\| e^{\left\|A_{2}\right\| h / M}\left\|B_{1}\right\|\right\}
\end{aligned}
$$

Furthermore, $K_{M j \mathrm{k} 1}$ has a uniform upper bound with respect to $M$ and $j$ defined as

$$
\begin{aligned}
& K_{\mathcal{C} \mathcal{A B k} 1}^{U}:=\frac{1}{2} h^{3} e^{\|A\| h}\left\|B_{1}\right\| K_{*} \\
& \cdot\left\{\left(\frac{1}{3}\left\|C_{0}\right\|+\left\|C_{0} A_{2}\right\| h\right)\|A\|^{2} e^{\|A\| h}+\left\|C_{0} A_{2}^{2}\right\| e^{\left\|A_{2}\right\| h}\right\}
\end{aligned}
$$

Lemmas 3 and 4 immediately lead to the following result.
Proposition 3: The inequality

$$
\left\|\mathcal{F}_{N M}^{-}-\mathcal{F}_{N M \mathrm{k} 1}^{-}\right\| \leq \frac{K_{M \mathrm{k} 1}}{M^{2}}
$$

holds where

$$
K_{M \mathrm{k} 1}:=K_{M \mathcal{D k} 1}+\sum_{j=0}^{N} K_{M j \mathrm{k} 1}
$$

In addition, $K_{M \mathrm{k} 1}$ has a uniform upper bound with respect to $M$ given by

$$
K_{\mathrm{k} 1}^{U}:=K_{\mathcal{D k} 1}^{U}+(N+1) K_{\mathcal{C} \mathcal{A B k} 1}^{U}
$$

We next provide a method for (exactly) computing $\left\|\mathcal{F}_{N M \mathrm{k} 1}^{-}\right\|$. By essentially the same arguments to those in [18],[21], we can have the following result.

Proposition 4: Let $V^{[0]}$ be the matrix consisting of the $L_{1}\left[0, h^{\prime}\right)$ norm of each entry of the matrix linear function $\overline{C_{0}} \Delta_{M 0} \overline{A_{d}^{\prime}\left(I-A \theta^{\prime}\right) B_{1}}$, while let $V^{\left[h^{\prime}\right]}$ be the matrix constructed in the same way from $\overline{C_{0}\left(I+A_{2} h^{\prime}\right)} \Delta_{M 0} \overline{A_{d}^{\prime}\left(I-A \theta^{\prime}\right) B_{1}}$. Furthermore, let $T_{j}^{[0]}(j=0, \cdots, N)$ be the matrix consisting of the $L_{1}\left[0, h^{\prime}\right)$ norm of each entry of the matrix linear function $\overline{C_{0}} \mathcal{A}_{M j} \overline{A_{d}^{\prime}\left(I-A \theta^{\prime}\right) B_{1}}$, while let $T_{j}^{\left[h^{\prime}\right]}(j=0, \cdots, N)$ be the matrix constructed in the same way from $\overline{C_{0}\left(I+A_{2} h^{\prime}\right)} \mathcal{A}_{M j} \overline{A_{d}^{\prime}\left(I-A \theta^{\prime}\right) B_{1}} . \quad$ Then, $\quad\left\|\mathcal{F}_{N M \mathrm{k} 1}^{-}\right\|$ coincides with the $\infty$-norm of the finite-dimensional matrix $F_{N M \mathrm{k} 1}^{-}$given by

$$
F_{N M \mathrm{k} 1}^{-}:=\left[\begin{array}{llllll}
\overline{D_{11}} & 0 & V^{[0]} & T_{0}^{[0]} & \cdots & T_{N}^{[0]} \\
\overline{D_{11}} & \overline{C_{1} B_{1} h^{\prime}} & V^{\left[h^{\prime}\right]} & T_{0}^{\left[h^{\prime}\right]} & \cdots & T_{N}^{\left[h^{\prime}\right]}
\end{array}\right]
$$

Combining the above propositions leads to the following second main result in this paper.

Theorem 2: The following inequality holds:

$$
\left\|F_{N M \mathrm{k} 1}^{-}\right\|-\frac{K_{M \mathrm{k} 1}}{M^{2}} \leq\left\|\mathcal{F}_{N}^{-}\right\| \leq\left\|F_{N M \mathrm{k} 1}^{-}\right\|+\frac{K_{M \mathrm{k} 1}}{M^{2}}
$$

This implies that upper and lower bounds of $\left\|\mathcal{F}_{N}^{-}\right\|$can be obtained through $\left\|F_{N M \mathrm{k} 1}^{-}\right\|$together with $K_{M \mathrm{k} 1} / M^{2}$, and by taking the fast-lifting parameter $M$ larger, the gap between those upper and lower bounds converges to 0 at no slower convergence rate than $1 / M^{2}$ (because $K_{M \mathrm{k} 1}$ has a uniform upper bound $K_{\mathrm{k} 1}^{U}$ ).

\section{Upper Bound of $\left\|\mathcal{F}_{N}^{+}\right\|$and Computation of $\|\mathcal{F}\|$}

In this subsection, we first remark that a computation method for an upper bound of $\left\|\mathcal{F}_{N}^{+}\right\|$has been given by the following proposition in [18]; note that the stability assumption of $\Sigma_{\mathrm{SD}}$ ensures the existence of $L$ such that $\left\|\mathcal{A}^{L}\right\|<1$.

Proposition 5 ([18], Proposition 3): If $\left\|\mathcal{A}^{L}\right\|<1$, then

$\left\|\mathcal{F}_{N}^{+}\right\| \leq \frac{\left\|C_{\Sigma} \mathcal{A}_{N L}\right\|}{1-\left\|\mathcal{A}^{L}\right\|}\left\|C_{0}\right\| e^{\left\|A_{2}\right\| h} h e^{\|A\| h} B_{1}=: K_{N L}$

where $\mathcal{A}_{N L}:=\left[\begin{array}{llll}\mathcal{A}^{N+1} & \mathcal{A}^{N+2} & \cdots & \mathcal{A}^{N+L}\end{array}\right]$, and $K_{N L}$ converges to 0 regardless of $L$ as $N \rightarrow \infty$.

By (19), this together with the results in the preceding subsection immediately leads to the following result, giving 
upper and lower bounds of $\|\mathcal{F}\|$ converging to each other as the parameters $M$ and $N$ tend to $\infty$.

Theorem 3: If $\left\|\mathcal{A}^{L}\right\|<1$, then

$$
\begin{aligned}
&\left\|F_{N M \mathrm{k} 0}^{-}\right\|-\frac{K_{M \mathrm{k} 0}}{M}-K_{N L} \leq\|\mathcal{F}\| \\
& \leq\left\|F_{N M \mathrm{k} 0}^{-}\right\|+\frac{K_{M \mathrm{k} 0}}{M}+K_{N L} \\
&\left\|F_{N M \mathrm{k} 1}^{-}\right\|-\frac{K_{M \mathrm{k} 1}}{M^{2}}-K_{N L} \leq\|\mathcal{F}\| \\
& \leq\left\|F_{N M \mathrm{k} 1}^{-}\right\|+\frac{K_{M \mathrm{k} 1}}{M^{2}}+K_{N L}
\end{aligned}
$$

Furthermore, $K_{M \mathrm{k} 0}$ and $K_{M \mathrm{k} 1}$ have uniform upper bounds $K_{\mathrm{k} 0}^{U}$ and $K_{\mathrm{k} 1}^{U}$ defined as (42) and (57), respectively, and $K_{M \mathrm{k} 0} / M$ and $K_{M \mathrm{k} 1} / M^{2}$ converges to 0 as $M \rightarrow \infty$, while $K_{N L}$ converges to 0 regardless of $L$ as $N \rightarrow \infty$.

Regarding how to take the parameters $N$ and $L$ as well as $M$, the guideline discussed in [18] applies as it is also to the kernel approximation approach developed in this paper.

We are in a position to discuss the effectiveness of the developed computation method for $\|\mathcal{F}\|$ with the kernel approximation approach, compared with the method in [18] through the input approximation approach. To this end, we remark that the input approximation approach developed in [18] leads to the inequality [18, Theorem 3]

$$
\begin{aligned}
\left\|F_{N M \mathrm{i} 0}^{-}\right\|-\frac{K_{M \mathrm{i} 0}}{M} & -K_{N L} \leq\|\mathcal{F}\| \\
& \leq\left\|F_{N M \mathrm{i} 0}^{-}\right\|+\frac{K_{M \mathrm{i} 0}}{M}+K_{N L} \\
\left\|F_{N M \mathrm{i} 1}^{-}\right\|-\frac{K_{M \mathrm{i} 1}}{M^{2}} & -K_{N L} \leq\|\mathcal{F}\| \\
& \leq\left\|F_{N M \mathrm{i} 1}^{-}\right\|+\frac{K_{M \mathrm{i} 1}}{M^{2}}+K_{N L}
\end{aligned}
$$

for the staircase and piecewise linear approximation schemes, respectively, with appropriately defined finite-dimensional matrices $F_{N M \mathrm{i} 0}^{-}$and $F_{N M \mathrm{i} 1}^{-}$and constants $K_{M \mathrm{i} 0}$ and $K_{M \mathrm{i} 1}$. It is important to remark that the constants $K_{M \mathrm{k} 0}$ and $K_{M \mathrm{k} 1}$ we have derived in this paper can be shown to be smaller than $K_{M \mathrm{i} 0}$ and $K_{M \mathrm{i} 1}$, respectively, given in [18] through parallel arguments. This implies that the kernel approximation approach can lead to a smaller gap between the upper and lower bound for the $L_{\infty}$-induced norm under the same parameter $M$ than the input approximation approach.

\section{CONCLUSION}

In this paper, we developed a new approach to computing the $L_{\infty}$-induced norm of sampled-data systems by using an idea of kernel approximation approach, which is stimulated by the success in computing the $L_{\infty}$-induced norm of a continuous-time system. Staircase and piecewise linear approximation schemes are applied via the fast-lifting treatment of sampled-data systems, so that the kernel function of the input operator and the hold function of the output operator associated with sampled-data systems are approximated by staircase or piecewise linear functions. We showed that upper and lower bounds of the $L_{\infty}$-induced norm can be readily computed through such approximation and that the gap between the upper bound and lower bound in the staircase approximation scheme or piecewise linear approximation scheme is ensured to converge to 0 at the rate of $1 / M$ or $1 / M^{2}$, respectively, where $M$ is the fast-lifting parameter. We also remarked that even though these convergence rates are qualitatively the same as those in the existing input approximation approach, the approximation errors through the new kernel approximation approach are smaller than those through the existing input approximation approach.

\section{REFERENCES}

[1] Y. Yamamoto, A function space approach to sampled data control systems and tracking problems, IEEE Trans. Automat. Contr., Vol. 39, No. 4, pp. 703-713 (1994).

[2] B. A. Bamieh and J. B. Pearson, A general framework for linear periodic systems with application to $H^{\infty}$ sampled-data systems, IEEE Trans. Automat. Contr., Vol. 37, No. 4, pp. 418-435 (1992).

[3] H. T. Toivonen, Sampled-data control of continuous-time systems with an $H_{\infty}$ optimality criterion, Automatica, Vol. 28 , No. 1 pp. $45-54$ (1992).

[4] B. D. O. Anderson and J. P. Keller, A new approach to the discretization of continuous-time controllers, IEEE Trans. Automat. Contr, Vol. 37, No. 2, pp. 214-223 (1992).

[5] Y. Yamamoto, A. G. Madievski and B. D. O. Anderson, Approximation of frequency response for sampled-data control systems, Automatica, Vol. 35, No. 4, pp. 729-734 (1999).

[6] M. Araki, Y. Ito and T. Hagiwara, Frequency response of sampled-data systems, Automatica, Vol. 32, No. 4, pp. 483-497 (1996).

[7] T. Hagiwara and M. Araki, FR-operator approach to the $H_{2}$ analysis and synthesis of sampled-data systems, IEEE Trans. Automat. Contr. Vol. 40, No. 8, pp. 1411-1421 (1995).

[8] L. Mirkin, H. Rotstein and Z. J. Palmor, $H_{2}$ and $H_{\infty}$ design of sampled-data systems using lifting. Part I: General framework and solutions, SIAM J. Control and Optimization, Vol. 38, No. 1, pp. 175196 (1999)

[9] L. Mirkin, H. Rotstein and Z. J. Palmor, $H_{2}$ and $H_{\infty}$ design of sampled-data systems using lifting. Part II: Properties of systems in the lifted domain, SIAM J. Control and Optimization, Vol. 38, No. 1, pp. 197-218 (1999).

[10] G. Tadmor, $H_{\infty}$ optimal sampled-data control in continuous time systems, Int. J. Control, Vol. 56, No. 1, pp. 99-141 (1992).

[11] T. Hagiwara and K. Okada, Modified fast-sample/fast-hold approximation and $\gamma$-independent $H_{\infty}$-discretisation for general sampled-data systems by fast-lifting, Int. J. Control, Vol. 82, No. 9, pp. 1762-1771 (2009).

[12] B. Bamieh and J. B. Pearson, The $H_{2}$ problem for sampled-data systems, Syst. Control Lett., Vol. 19, No. 1, pp. 1-12 (1992).

[13] T. Chen and B. A. Francis, $\mathrm{H}_{2}$-optimal sampled-data control, IEEE Trans. Automat. Contr., Vol. 36, No.4, pp. 387-397 (1991).

[14] P. P. Khargonekar and N. Sivashankar, $\mathrm{H}_{2}$ optimal control for sampled-data systems, Syst. Control Lett., Vol. 17, No. 6, pp. 425436 (1991).

[15] N. Sivashankar and P. P. Khargonekar, Induced norms for sampled-data systems, Automatica, Vol. 28, No. 6, pp. 1267-1272 (1992).

[16] B. A. Bamieh, M. A. Dahleh and J. B. Pearson, Minimization of the $L^{\infty}$-induced norm for sampled-data systems, IEEE Trans. Automat. Contr., Vol. 38, No. 5, pp. 717-732 (1993).

[17] G. E. Dullerud and B. A. Francis, $L_{1}$ analysis and design of sampleddata systems, IEEE Trans. Automat. Contr., Vol. 37, No. 4 pp. 436-446 (1992).

[18] J. H. Kim and T. Hagiwara, $L_{\infty}$-induced norm analysis of sampleddata systems via piecewise constant and linear approximations, Automatica, Vol. 51, No. 1, pp. 223-232 (2015).

[19] T. Hagiwara and H. Umeda, Modified fast-sample/fast-hold approximation for sampled-data system analysis, Eur. J. Control, Vol. 14, No. 4, pp. 286-296 (2008).

[20] J. H. Kim and T. Hagiwara, Computing the $L_{\infty}[0, h)$-induced norm of a compression operator via fast-lifting, Syst. Control Lett., Vol. 67 , May, pp. 1-8 (2014).

[21] J. H. Kim and T. Hagiwara, Computing the $L_{\infty}$-induced norm of LTI systems via kernel approximation and its comparison with input approximation, IET Control Theory Appl., accepted for publication. 